\title{
SLICE MONOGENIC FUNCTIONS OF A CLIFFORD VARIABLE VIA THE $S$-FUNCTIONAL CALCULUS
}

\author{
FABRIZIO COLOMBO, DAVID P. KIMSEY, STEFANO PINTON, AND IRENE SABADINI
}

(Communicated by Javad Mashreghi)

\begin{abstract}
In this paper we define a new function theory of slice monogenic functions of a Clifford variable using the $S$-functional calculus for Clifford numbers. Previous attempts of such a function theory were obstructed by the fact that Clifford algebras, of sufficiently high order, have zero divisors. The fact that Clifford algebras have zero divisors does not pose any difficulty whatsoever with respect to our approach. The new class of functions introduced in this paper will be called the class of slice monogenic Clifford functions to stress the fact that they are defined on open sets of the Clifford algebra $\mathbb{R}_{n}$. The methodology can be generalized, for example, to handle the case of noncommuting matrix variables.
\end{abstract}

\section{INTRODUCTION}

This paper is inspired by recent advances in the spectral theory on the $S$ spectrum for Clifford operators in [7, where fully Clifford operators play a crucial role in the approach. These new developments in operator theory have deep consequences on the function theory of slice monogenic functions because they highlight properties and potentialities of the Cauchy formula of slice monogenic functions that have impact on future researches.

In the literature, the various hyperholomorphic function theories for Clifford algebra valued functions mainly consider smooth functions defined on an open set $U$ in the Euclidean space $\mathbb{R}^{n+1}$ and not in the whole Clifford algebra $\mathbb{R}_{n}$ (we denote by $\mathbb{R}_{n}$ the Clifford algebra over $n$ imaginary units $e_{i}, e_{i}^{2}=-1$ ).

When the hyperholomorphic functions with values in a Clifford algebra, or, more in general, in an associative algebra were introduced, no restrictions were imposed on the domain; see e.g. 27,32 and references therein. However, it was soon realized that the presence of zero divisors in the domain could complicate the analysis of the hyperholomorphic functions; see e.g. 31]. Thus, the problem of treating a function theory on more general domains in the algebra remained unsolved, a part the case of bicomplex numbers; see 29] and the references therein.

The more recent theory of slice hyperholomorphic functions started in the quaternionic case with the paper [20]. Then it was first generalized to the case of functions with values in a Clifford algebra, see [11,13, 14, which were further studied in

Received by the editors January 13, 2021, and, in revised form, May 10, 2021.

2020 Mathematics Subject Classification. Primary 47A10, 47A60.

Key words and phrases. Slice monogenic functions, noncommuting matrix variables, $S$ functional calculus, $S$-spectrum.

The first author was partially supported by the PRIN project Direct and inverse problems for partial differential equations: theoretical aspects and applications. 
[8, 15, 30, in the algebra of octonions [21, and also to the case of a real alternative algebra [22, using however a different, although related, definition.

Later, other variations of the notion of slice hyperholomorphicity were introduced; see [10, 18, 25, 26]; however all of them have in common the fact that the domain of the functions can be expressed as the union of complex planes. In the particular case of Clifford algebras, this means that one cannot consider a fully Clifford variable as input of a function.

The function theory of slice hyperholomorphic functions was developed under the need of providing all the necessary tools to develop the so-called $S$-functional calculus for $n$-tuples of operators, see [2,12,16, 19] and [15, which was defined for paravector operators and was based on the Cauchy formula for slice monogenic functions and on the $S$-spectrum.

In 2020 the first and second authors proved the spectral theorem for fully Clifford operators based on the $S$-spectrum in [7. The fact that the spectral theorem exists in such a general setting gives a strong motivation to consider the $S$-functional calculus for fully Clifford operators and, more generally, also for operators acting on a two-sided modules over more general algebras. In fact, in [6] it is shown that $S$-functional calculus and its properties can be extended to fully Clifford operators or more general operators. The fact the $S$-spectrum is defined for operators acting on two sided modules over a real alternative algebra (which includes all Clifford algebras of the form $\mathbb{R}_{n}$ ) and that the basic properties remain intact (i.e., the $S$ spectrum of a bounded operator is a non-empty compact set) was observed in [23] and used for analysis of semigroups.

The main novelty of this paper is to use the spectral theory on the $S$-spectrum to define slice monogenic functions of a Clifford variable. The strategy is general and can be used in other cases that we shall discuss in the last section of the paper. We point out that the idea of using operator theory to obtain results in function theory is not new. In fact, several results for noncommuting variables are obtained via the Taylor functional calculus; see the book 24] for further discussions.

To explain how the strategy based on the $S$-spectrum works, we first make some observations on the Cauchy formulas of the theory of several complex variables and of monogenic functions. Then we compare these two formulas with the Cauchy formula of slice monogenic functions and we show the consequences on the function theories.

We recall that the holomorphic Cauchy kernel

$$
\left(\lambda_{1}, \ldots, \lambda_{n}\right) \mapsto \prod_{j=1}^{n}\left(\lambda_{j}-z_{j}\right)^{-1}
$$

is defined in $\mathbb{C}^{n} \backslash\left\{\left(z_{1}, \ldots, z_{n}\right)\right\}$ and the Cauchy formula for holomorphic functions in $n$ complex variables $z_{1}, \ldots, z_{n}$ is given by

$$
f\left(z_{1}, \ldots, z_{n}\right)=\frac{1}{(2 \pi i)^{n}} \int_{C_{1}} \ldots \int_{C_{n}} \prod_{j=1}^{n}\left(\lambda_{j}-z_{j}\right)^{-1} f\left(\lambda_{1}, \ldots, \lambda_{n}\right) d \lambda,
$$

where $d \lambda=d \lambda_{1} \cdots d \lambda_{n}$ and $f$ is any holomorphic function in a neighbourhood of the point $\left(z_{1}, \ldots, z_{n}\right) \in \mathbb{C}^{n}$. For each $j=1, \ldots, n$ the simple closed contour $C_{j}$ surrounds $z_{j}$ and $C_{1} \times \ldots \times C_{n}$ is contained in the domain of $f$ in $\mathbb{C}^{n}$. It is clear that in this formula one can form functions of $n$-tuples operators $A_{j}$ for $j=1, \ldots, n$, by 
replacing $\lambda_{j}-z_{j}$ by $\lambda_{j} \mathcal{I}-A_{j}$. Since $\lambda_{j}$ and $z_{j}$ are complex numbers, then $A_{j}$, for $j=1, \ldots, n$, have to be complex operators.

Let us now consider another higher dimensional generalization, namely one of hyperholomorphic functions. Let $\mathbb{R}_{n}$ be the real Clifford algebra over $n$ imaginary units $e_{1}, \ldots, e_{n}$ satisfying the relations $e_{\ell} e_{m}+e_{m} e_{\ell}=0, \ell \neq m, e_{\ell}^{2}=-1$. If $U \subseteq \mathbb{R}^{n+1}$ is an open set, a function $f: U \subseteq \mathbb{R}^{n+1} \rightarrow \mathbb{R}_{n}$ can be interpreted as a function of the paravector $x=x_{0}+e_{1} x_{1}+\ldots+e_{n} x_{n}$. The monogenic Cauchy kernel, see [3, 17, is

$$
G_{s}(x):=\frac{1}{\sigma_{n}}(\overline{s-x})\left(\sum_{j=0}^{n}\left(s_{j}-x_{j}\right)^{2}\right)^{-\frac{n+1}{2}}, \quad x, s \in \mathbb{R}^{n+1}, \quad x \neq s,
$$

where $\sigma_{n}:=2 \pi^{\frac{n+1}{2}} / \Gamma\left(\frac{n+1}{2}\right)$ is the volume of unit sphere in $\mathbb{R}^{n+1}$. Let $f$ be a left monogenic function on an open set that contains $\bar{U}$; then the Cauchy formula

$$
f(x)=\int_{\partial U} G_{s}(x) \eta(s) f(s) d S(s)
$$

holds, for every $x$ in $U$, where $U$ is an open set in $\mathbb{R}^{n+1}$ with smooth boundary $\partial U, \eta(s)$ is the outer unit normal to $\partial U$ and $d S(s)$ is the scalar element of surface area on $\partial U$. Also in this case, the Cauchy kernel contains the difference of the coordinates $s_{j}-x_{j}$ so to define a functional calculus, for consistency, the differences $s_{j}-x_{j}$ can be replaced by the operators $s_{j} \mathcal{I}-T_{j}$, where $T_{j}$ are real operators with real spectrum. It is unclear how to give a meaning to the monogenic Cauchy formula (1.2) when we suppose to replace the variable $x$ by a paravector operator $T=T_{0}+e_{1} T_{1}+\cdots+e_{n} T_{n}$ or, more in general, by a fully Clifford operator. The same problem occurs also with formula (1.1) which cannot work for such operators.

The Cauchy formula for slice monogenic function has a greater flexibility because the paravector variables $s$ and $x$, appearing in the slice monogenic Cauchy kernel, play different roles. Consider the left slice monogenic Cauchy kernel

$$
S_{L}^{-1}(s, x):=-\left(x^{2}-2 \operatorname{Re}(s) x+|s|^{2}\right)^{-1}(x-\bar{s}),
$$

where $x, s \in \mathbb{R}^{n+1}$, and $x \notin[s]$ (see Section 2 for the notations) are paravectors. From a heuristic point of view, we see that the variable $x$ appears with a different role with respect to the variable $s$ and this is clearly visible if one is willing to replace $x$ or $s$ by an operator $T$. In the case of $s$, we have to give meaning to $\operatorname{Re}(s)$ and to $|s|^{2}$ in terms of the operator $T$. But with respect to $x$ we only have to give meaning to powers of $T$, in fact only the square of $T$. Any mathematical object $T$ whose powers have a meaning is a possible candidate for the replacement. In the original version of the $S$-functional calculus the paravector $x$ is replaced by a paravector operator $T=T_{0}+T_{1} e_{1}+\cdots+T_{n} e_{n}$ with not necessarily commuting components $T_{j}, j=0, \ldots, n$.

The functional calculus for fully Clifford operators opens the way to define slice monogenic functions of a Clifford variable $\hat{x} \in \mathbb{R}_{n}$ using the slice monogenic Cauchy formula. To this end, we define the $S$-spectrum of the Clifford number $\hat{x}$ as

$$
\sigma_{S}(\hat{x})=\left\{s \in \mathbb{R}^{n+1}: \hat{x}^{2}-2 \operatorname{Re}(s) \hat{x}+|s|^{2} \quad \text { is not invertible in } \mathbb{R}_{n}\right\} .
$$

Now let $\hat{x} \in \mathbb{R}_{n}$ and let $U \subset \mathbb{R}^{n+1}$ be a bounded slice Cauchy domain that contains $\sigma_{S}(\hat{x})$ and for $\mathrm{j} \in \mathbb{S}\left(\mathbb{S}\right.$ is the sphere of paravectors $s$ with $s_{0}=0, s^{2}=-1$ ) we set $d s_{\mathrm{j}}=d s(-\mathrm{j})$. Assume that $f$ is a (left) slice monogenic function on a set that 
contains $\bar{U}$ and assume that $U$ contains the $S$-spectrum of $\hat{x}$. We define the (left) slice monogenic function of the Clifford variable $\hat{x}$ as

$$
f(\hat{x}):=\frac{1}{2 \pi} \int_{\partial\left(U \cap \mathbb{C}_{\mathrm{j}}\right)} S_{L}^{-1}(s, \hat{x}) d s_{\mathrm{j}} f(s) .
$$

The function $f(\hat{x})$ is well defined because then the integral (1.3) depends neither on $U$ nor on the imaginary unit $\mathrm{j} \in \mathbb{S}$. Observe that in the case $\hat{x}$ is a paravector then the definition (1.3) becomes the Cauchy formula for slice monogenic functions.

When $\hat{x}$ varies in a set $W$ contained in $\mathbb{R}_{n}$, the formula gives a function of $\hat{x}$ since $U$ is chosen sufficiently large such that it contains $\sigma_{S}(\hat{x})$ for all $\hat{x} \in W$. A similar definition holds in more general cases, for example, in the case of matrix variables.

\section{Preliminary Results}

In this section we collect the preliminary results which are needed in the sequel. An element in the Clifford algebra $\mathbb{R}_{n}$ will be denoted by $\hat{x}=\sum_{A} e_{A} x_{A}$, with $x_{A} \in \mathbb{R}$, where $A=\left\{\ell_{1} \ldots \ell_{r}\right\} \in \mathcal{P}\{1,2, \ldots, n\}, \quad \ell_{1}<\ldots<\ell_{r}$ is a multi-index and $e_{A}=e_{\ell_{1}} e_{\ell_{2}} \ldots e_{\ell_{r}}, e_{\emptyset}=1$. An element $\left(x_{0}, x_{1}, \ldots, x_{n}\right) \in \mathbb{R}^{n+1}$ will be identified with the element $x=x_{0}+\underline{x}=x_{0}+\sum_{\ell=1}^{n} x_{\ell} e_{\ell} \in \mathbb{R}_{n}$ and will be called a paravector and the real part $x_{0}$ of $x$ will also be denoted by $\operatorname{Re}(x)$. The norm of $x \in \mathbb{R}^{n+1}$ is defined as $|x|^{2}=x_{0}^{2}+x_{1}^{2}+\ldots+x_{n}^{2}$. More generally the norm of $\hat{x}$ is given by $|\hat{x}|^{2}=\sum_{A}\left|x_{A}\right|^{2}$ and is called the Euclidean norm. The conjugate of $x$ is defined by $\bar{x}=x_{0}-\underline{x}=x_{0}-\sum_{\ell=1}^{n} x_{\ell} e_{\ell}$. With a slight abuse of notation if $x \in \mathbb{R}_{n}$ is a paravector, then we will write $x \in \mathbb{R}^{n+1}$.

We denote by $\mathbb{S}$ the sphere

$$
\mathbb{S}=\left\{\underline{x}=e_{1} x_{1}+\ldots+e_{n} x_{n}: x_{1}^{2}+\ldots+x_{n}^{2}=1\right\} .
$$

Note that for $\mathrm{j} \in \mathbb{S}$ we obviously have $\mathrm{j}^{2}=-1$. Given an element $x=x_{0}+\underline{x} \in \mathbb{R}^{n+1}$ let us set $\mathrm{j}_{x}=\underline{x} /|\underline{x}|$ if $\underline{x} \neq 0$, and given an element $x \in \mathbb{R}^{n+1}$, the set

$$
[x]:=\left\{y \in \mathbb{R}^{n+1}: y=x_{0}+\mathrm{j}|\underline{x}|, \mathrm{j} \in \mathbb{S}\right\}
$$

is an $(n-1)$-dimensional sphere in $\mathbb{R}^{n+1}$. The vector space $\mathbb{R}+\mathrm{j} \mathbb{R}$ passing through 1 and $\mathrm{j} \in \mathbb{S}$ will be denoted by $\mathbb{C}_{\mathrm{j}}$ and an element belonging to $\mathbb{C}_{\mathrm{j}}$ will be indicated by $u+\mathrm{j} v$, for $u, v \in \mathbb{R}$.

We recall the definition of slice monogenic functions which is slightly different from the original one; this definition allows us to define functions on axially symmetric domains that do not necessarily intersect the real axis. The proofs are minor modifications of the ones in [15].

Definition 2.1. Let $U \subseteq \mathbb{R}^{n+1}$. We say that $U$ is axially symmetric if $[x] \in U$ for every $x \in U$.

Definition 2.2 is nowadays systematically used in operator theory, see [4, 5], and also for vector-valued operator functions.

Definition 2.2 (Slice monogenic functions). Let $U \subseteq \mathbb{R}^{n+1}$ be an axially symmetric open set and let $\mathcal{U}=\left\{(u, v) \in \mathbb{R}^{2} \mid u+\mathbb{S} v \subseteq U\right\}$. A function $f: U \rightarrow \mathbb{R}_{n}$ is called a left slice function, if it is of the form

$$
f(x)=f_{0}(u, v)+\mathrm{j} f_{1}(u, v) \quad \text { for } x=u+\mathrm{j} v \in U
$$


with two functions $f_{0}, f_{1}: \mathcal{U} \rightarrow \mathbb{R}_{n}$ that satisfy the compatibility conditions

$$
f_{0}(u,-v)=f_{0}(u, v), \quad f_{1}(u,-v)=-f_{1}(u, v) .
$$

If in addition $f_{0}$ and $f_{1}$ satisfy the Cauchy-Riemann-equations

$$
\begin{aligned}
& \frac{\partial}{\partial u} f_{0}(u, v)-\frac{\partial}{\partial v} f_{1}(u, v)=0 \\
& \frac{\partial}{\partial v} f_{0}(u, v)+\frac{\partial}{\partial u} f_{1}(u, v)=0
\end{aligned}
$$

then $f$ is called left slice monogenic. A function $f: U \rightarrow \mathbb{R}_{n}$ is called a right slice function if it is of the form

$$
f(x)=f_{0}(u, v)+f_{1}(u, v) \mathrm{j} \quad \text { for } x=u+\mathrm{j} v \in U
$$

with two functions $f_{0}, f_{1}: \mathcal{U} \rightarrow \mathbb{R}_{n}$ that satisfy (2.1). If in addition $f_{0}$ and $f_{1}$ satisfy the Cauchy-Riemann-equations, then $f$ is called right slice monogenic.

Definition 2.3. If $f$ is a left (or right) slice function such that $f_{0}$ and $f_{1}$ are real-valued, then $f$ is called intrinsic. We denote the sets of left and right slice monogenic functions on $U$ by $\mathcal{S M}_{L}(U)$ and $\mathcal{S} \mathcal{M}_{R}(U)$, respectively. The set of intrinsic slice monogenic functions on $U$ will be denoted by $\mathcal{N}(U)$.

Definition 2.4. Let $x, s \in \mathbb{R}^{n+1}$ with $x \notin[s]$. We define the left slice monogenic Cauchy kernel $S_{L}^{-1}(s, x)$ as

$$
S_{L}^{-1}(s, x):=-\left(x^{2}-2 \operatorname{Re}(s) x+|s|^{2}\right)^{-1}(x-\bar{s}),
$$

and the right slice monogenic Cauchy kernel $S_{R}^{-1}(s, x)$ as

$$
S_{R}^{-1}(s, x):=-(x-\bar{s})\left(x^{2}-2 \operatorname{Re}(s) x+|s|^{2}\right)^{-1} .
$$

The following results are well known.

Lemma 2.5. Let $x, s \in \mathbb{R}^{n+1}$ with $s \notin[x]$. The left slice hyperholomorphic Cauchy kernel $S_{L}^{-1}(s, x)$ is left slice hyperholomorphic in $x$ and right slice hyperholomorphic in s. The right slice hyperholomorphic Cauchy kernel $S_{R}^{-1}(s, x)$ is left slice hyperholomorphic is $s$ and right slice hyperholomorphic in $x$.

Definition 2.6 (Slice Cauchy domain). An axially symmetric open set $U \subset \mathbb{R}^{n+1}$ is called a slice Cauchy domain if $U \cap \mathbb{C}_{\mathrm{j}}$ is a Cauchy domain in $\mathbb{C}_{\mathrm{j}}$ for any $\mathrm{j} \in \mathbb{S}$. More precisely, $U$ is a slice Cauchy domain if, for any $\mathrm{j} \in \mathbb{S}$, the boundary $\partial\left(U \cap \mathbb{C}_{\mathbf{j}}\right)$ of $U \cap \mathbb{C}_{\mathrm{j}}$ is the union of a finite number of non-intersecting piecewise continuously differentiable Jordan curves in $\mathbb{C}_{\mathrm{j}}$.

Theorem 2.7 (The Cauchy formulas). Let $U \subset \mathbb{R}^{n+1}$ be a bounded slice Cauchy domain, let $\mathrm{j} \in \mathbb{S}$ and set $d s_{\mathrm{j}}=d s(-\mathrm{j})$. If $f$ is a (left) slice hyperholomorphic function on a set that contains $\bar{U}$ then

$$
f(x)=\frac{1}{2 \pi} \int_{\partial\left(U \cap \mathbb{C}_{\mathrm{j}}\right)} S_{L}^{-1}(s, x) d s_{\mathrm{j}} f(s), \quad \text { for any } \quad x \in U .
$$

If $f$ is a right slice hyperholomorphic function on a set that contains $\bar{U}$, then

$$
f(x)=\frac{1}{2 \pi} \int_{\partial\left(U \cap \mathbb{C}_{\mathrm{j}}\right)} f(s) d s_{\mathrm{j}} S_{R}^{-1}(s, x), \quad \text { for any } \quad x \in U .
$$

The integrals (2.4) and (2.5) depend neither on $U$ nor on the imaginary unit $\mathrm{j} \in \mathbb{S}$. 


\section{Slice monogenic functions of a Clifford variable}

Using the results in the previous section, we can now define monogenic function of a Clifford variable that is not necessarily a paravector. We start with some examples considering a slice monogenic polynomial $P(x)=\sum_{m=0}^{M} x^{m} a_{m}, \quad a_{m} \in \mathbb{R}_{n}$ of order $M$. We can define the slice monogenic polynomial of the Clifford number $\hat{x} \in \mathbb{R}_{n}$ by simply replacing the paravector $x$ by $\hat{x}$ and we get $P(\hat{x})=\sum_{m=0}^{M} \hat{x}^{m} a_{m}, \quad a_{m} \in \mathbb{R}_{n}$. In the case we consider a power series expansion of a slice monogenic function $f$ that converges in a suitable ball centered at the origin, replacing $x$ by $\hat{x}$ we get $f(\hat{x})=\sum_{m=0}^{+\infty} \hat{x}^{m} a_{m}, \quad a_{m} \in \mathbb{R}_{n}$ and $f(\hat{x})$ is well defined for those Clifford numbers $\hat{x}$ such that the series is absolutely convergent. If $x=x_{0}+x_{1} e_{1}+\cdots+x_{n} e_{n}$ and $s=x_{0}+s_{1} e_{1}+\cdots+s_{n} e_{n}$ are paravectors, then the Cauchy kernels are expressed in power series as

$$
S_{L}^{-1}(s, x):=\sum_{m=0}^{+\infty} x^{m} s^{-1-m}, \quad S_{R}^{-1}(s, x):=\sum_{m=0}^{+\infty} s^{-1-m} x^{m}, \quad|x|<|s| .
$$

Below we shall make use of the norm $|\hat{x}|_{1}$ defined by

$$
|\hat{x}|_{1}=\sum_{A}\left|x_{A}\right| \text {. }
$$

It is equivalent to the Euclidean norm $|\hat{x}|$ but more convenient in some circumstances. In fact, for the norm $|\hat{x}|_{1}$ we have that $\left|\hat{x}^{m}\right|_{1} \leq|\hat{x}|_{1}^{m}$ while for the Euclidean norm there is a constant $C \geq 1$ such that $\left|\hat{x}^{m}\right| \leq C^{m}|\hat{x}|^{m}$. In order to avoid the constant $C$ we will use the norm $|\cdot|_{1}$, for fully Clifford numbers, and we write $|\cdot|$ instead of $|\cdot|_{1}$ when no confusion arises.

Now observe that we can define the $S$-resolvent functions associated with the Clifford number $\hat{x} \in \mathbb{R}_{n}$ as follows.

Definition 3.1. Let $\hat{x} \in \mathbb{R}_{n}$ and let $s \in \mathbb{R}^{n+1}$. We define the left and the right $S$-resolvent series associated with $\hat{x} \in \mathbb{R}_{n}$ as follows:

$$
S_{L}^{-1}(s, \hat{x}):=\sum_{m=0}^{+\infty} \hat{x}^{m} s^{-1-m}, \quad S_{R}^{-1}(s, \hat{x}):=\sum_{m=0}^{+\infty} s^{-1-m} \hat{x}^{m} .
$$

Theorem 3.2. Let $\hat{x} \in \mathbb{R}_{n}$ and let $s \in \mathbb{R}^{n+1}$ be such that $|\hat{x}|_{1}<|s|$. Then the left and the right $S$-resolvent series associated with $\hat{x} \in \mathbb{R}_{n}$ are absolutely convergent.

Proof. Observe that when $b$ is a paravector and $\hat{x}$ is a Clifford number, for the Euclidean norm, we have

$$
|\hat{x} b|=|\hat{x}||b| .
$$

So we have that $\left|\hat{x}^{m} s^{-1-m}\right|=\left|\hat{x}^{m}\right||s|^{-1-m}$ because the inverse of a paravector is still a paravector. Finally, we get

$$
\left|\hat{x}^{m} s^{-1-m}\right|=\left|\hat{x}^{m}\right||s|^{-1-m} \leq C\left|\hat{x}^{m}\right|_{1}|s|^{-1-m} \leq C|\hat{x}|_{1}^{m}|s|^{-1-m} .
$$

The proof follows from the convergence of the geometric series.

Theorem 3.3. Let $\hat{x} \in \mathbb{R}_{n}$ and let $s \in \mathbb{R}^{n+1}$ be such that $|\hat{x}|_{1}<|s|$. Then we have

$$
\left(\hat{x}^{2}-2 s_{0} \hat{x}+|s|^{2}\right)^{-1}=\sum_{m=0}^{+\infty} \hat{x}^{m} \sum_{k=0}^{m}(\bar{s})^{-k-1} s^{-m+k-1} .
$$


Proof. The proof follows standard techniques; see e.g. the proof of Theorem 3.1.5 in the book $[5$.

Theorem 3.4 shows that, when we replace the paravector $x$ by the Clifford number $\hat{x}$ in the Cauchy kernel expansion, the sum of the series is formally obtained by replacing $x$ by $\hat{x}$ in the Cauchy kernel.

Theorem 3.4. Let $\hat{x} \in \mathbb{R}_{n}, s \in \mathbb{R}^{n+1}$. Then, for $|\hat{x}|_{1}<|s|$ we have

$$
\sum_{m=0}^{+\infty} \hat{x}^{m} s^{-1-m}=-\left(\hat{x}^{2}-2 \operatorname{Re}(s) \hat{x}+|s|^{2}\right)^{-1}(\hat{x}-\bar{s}),
$$

and

$$
\sum_{m=0}^{+\infty} s^{-1-m} \hat{x}^{m}=-(\hat{x}-\bar{s})\left(\hat{x}^{2}-2 \operatorname{Re}(s) \hat{x}+|s|^{2}\right)^{-1} .
$$

Proof. We show just (3.1) since the other case follows with a similar argument, which is the one used in [15]; in fact it is enough to show the identity

$$
\bar{s}-\hat{x}=\left(\hat{x}^{2}-2 \operatorname{Re}(s) \hat{x}+|s|^{2}\right) \sum_{m=0}^{+\infty} \hat{x}^{m} s^{-1-m}
$$

because $\hat{x}^{2}-2 \operatorname{Re}(s) \hat{x}+|s|^{2}$ is invertible by Theorem 3.3 . Since $s$ is a paravector the relations $2 \operatorname{Re}(s)=s+\bar{s}$ and $|s|^{2}=s \bar{s}=\bar{s} s$ are real and hence they commute with the Clifford number $\hat{x}$, we get

$$
\begin{aligned}
\left(\hat{x}^{2}-2 \operatorname{Re}(s) \hat{x}+|s|^{2}\right) \sum_{m=0}^{+\infty} \hat{x}^{m} s^{-m-1} & \\
\quad= & \sum_{m=1}^{+\infty} \hat{x}^{m+1} s^{-m}-\sum_{n=0}^{+\infty} \hat{x}^{m+1} s^{-m}-\sum_{m=0}^{+\infty} \hat{x}^{m+1} s^{-m-1} \bar{s}+\sum_{m=0}^{+\infty} \hat{x}^{m} s^{-m} \bar{s} \\
& =\bar{s}-\hat{x} .
\end{aligned}
$$

It is now natural to define the $S$-spectrum and the $S$-resolvent set of a Clifford number $\hat{x} \in \mathbb{R}_{n}$ (cf. [23]).

Definition 3.5. Let $\hat{x} \in \mathbb{R}_{n}, s \in \mathbb{R}^{n+1}$. We define the $S$-spectrum of the Clifford number $\hat{x} \in \mathbb{R}_{n}$ as

$$
\sigma_{S}(\hat{x})=\left\{s \in \mathbb{R}^{n+1}: \hat{x}^{2}-2 \operatorname{Re}(s) \hat{x}+|s|^{2} \quad \text { is not invertible in } \mathbb{R}_{n}\right\}
$$

and the $S$-resolvent set as

$$
\rho_{S}(\hat{x})=\mathbb{R}^{n+1} \backslash \sigma_{S}(\hat{x}) .
$$

Example 3.6. Let us consider the Clifford algebra $\mathbb{R}_{n}$ : if $\hat{x}=e_{A}$ and $e_{A}^{2}=1$ then we have that $1-2 \operatorname{Re}(s) \hat{x}+|s|^{2}$ is not invertible if and only if $s= \pm 1$ and so $\sigma_{S}\left(e_{A}\right)=\{ \pm 1\}$. If $e_{A}^{2}=-1$, then $-1-2 \operatorname{Re}(s) \hat{x}+|s|^{2}$ is not invertible if and only if $\operatorname{Re}(s)=0$ and $|s|=1$ so that $\sigma_{S}\left(e_{A}\right)=\mathbb{S}$.

We now consider the case of $\mathbb{R}_{3}$. Setting $\omega_{ \pm}=\frac{1}{2}\left(1 \pm e_{123}\right)$ we have that any element in the algebra can be written as $\hat{x}=\omega_{+} q_{+}+\omega_{-} q_{-}$where $q_{ \pm}$are quaternions belonging to the algebra $\mathbb{H}$ with generators $e_{1}, e_{2}$ and $\omega_{ \pm}$are two idempotents such that $\omega_{+}+\omega_{-}=1$. As it is well known and easily verified, the zero divisors are 
quaternionic multiples of $\omega_{+}$or $\omega_{-}$. Thus $\omega_{ \pm}^{2}-2 \operatorname{Re}(s) \omega_{ \pm}+|s|^{2}$ is not invertible for $s=0,1$ so $\sigma_{S}\left(\omega_{ \pm}\right)=\{0,1\}$.

In general, we have:

Theorem 3.7 (Structure of the $S$-spectrum). Let $\hat{x} \in \mathbb{R}_{n}$. Then $\sigma_{S}(\hat{x})$ and $\rho_{S}(\hat{x})$ are axially symmetric sets in $\mathbb{R}^{n+1}$.

Proof. It is an immediate consequence of the definition.

Definition 3.8 ( $S$-resolvent functions of $\hat{x}$ ). Let $\hat{x} \in \mathbb{R}_{n}$ and $s \in \rho_{S}(\hat{x})$. We define the left $S$-resolvent functions associated with the Clifford number $\hat{x}$ as

$$
S_{L}^{-1}(s, \hat{x}):=-\left(\hat{x}^{2}-2 \operatorname{Re}(s) \hat{x}+|s|^{2}\right)^{-1}(\hat{x}-\bar{s})
$$

and the right $S$-resolvent functions associated with the Clifford number $\hat{x}$ as

$$
S_{R}^{-1}(s, \hat{x}):=-(\hat{x}-\bar{s})\left(\hat{x}^{2}-2 \operatorname{Re}(s) \hat{x}+|s|^{2}\right)^{-1} .
$$

Observe that the $S$-resolvent functions are slice monogenic with respect to the variable $s$ for all $s \in \rho_{S}(\hat{x})$, cfr. Lemma 2.5, but it is not slice monogenic in $\hat{x}$.

Lemma 3.9. Let $\hat{x} \in \mathbb{R}_{n}$. Then the left $S$-resolvent function $S_{L}^{-1}(s, \hat{x})$ is right slice monogenic function of the variable $s$ on $\rho_{S}(\hat{x})$ and the right $S$-resolvent function $S_{R}^{-1}(s, \hat{x})$ is a left slice monogenic function of the variable $s$ on $\rho_{S}(\hat{x})$.

Proof. The proof follows by direct computations.

Theorem 3.10. Let $s \in \mathbb{R}^{n+1}, a \in \mathbb{R}_{n}, \ell \in \mathbb{N} \cup\{0\}$ and consider the monomial $s^{\ell} a$. Let $\hat{x} \in \mathbb{R}_{n}$ and $\sigma_{S}(\hat{x}) \subset U \subset \mathbb{R}^{n+1}$ where $U$ is a bounded slice Cauchy domain. Then, for every choice of $\mathrm{j} \in \mathbb{S}$, we have

$$
\hat{x}^{\ell} a=\frac{1}{2 \pi} \int_{\partial\left(U \cap \mathbb{C}_{\mathrm{j}}\right)} S_{L}^{-1}(s, \hat{x}) d s_{\mathrm{j}} s^{\ell} a,
$$

and

$$
a \hat{x}^{\ell}=\frac{1}{2 \pi} \int_{\partial\left(U \cap \mathbb{C}_{\mathrm{j}}\right)} a s^{\ell} d s_{\mathrm{j}} S_{R}^{-1}(s, \hat{x}) .
$$

Proof. We just consider (3.3) since (3.4) follows in a similar way. Let us consider the power series expansion of the $S$-resolvent function $S_{L}^{-1}(s, \hat{x})$ and assume that $U$ is a ball $B_{r}(0)$ centered in the origin, with radius $r>|\hat{x}|_{1}$, so we have

$$
\frac{1}{2 \pi} \int_{\partial\left(B_{r}(0) \cap \mathbb{C}_{\mathrm{j}}\right)} S_{L}^{-1}(s, \hat{x}) d s_{\mathrm{j}} s^{\ell} a=\frac{1}{2 \pi} \sum_{m=0}^{+\infty} \hat{x}^{m} \int_{\partial\left(B_{r}(0) \cap \mathbb{C}_{\mathrm{j}}\right)} s^{-1-m+\ell} d s_{\mathrm{j}} a .
$$

Since

$$
\int_{\partial\left(B_{r}(0) \cap \mathbb{C}_{\mathrm{j}}\right)} d s_{\mathrm{j}} s^{-m-1+\ell}=2 \pi \delta_{m, \ell},
$$

where $\delta_{m, \ell}$ is the Kronecker delta, and, by the Cauchy theorem, the above integrals are not affected if we replace $B_{r}(0)$ by $U$, for any $\mathrm{j} \in \mathbb{S}$, we have

$$
\frac{1}{2 \pi} \sum_{m=0}^{+\infty} \hat{x}^{m} \int_{\partial\left(B_{r}(0) \cap \mathbb{C}_{\mathrm{j}}\right)} s^{-1-m+\ell} d s_{\mathrm{j}} a=\frac{1}{2 \pi} \sum_{m=0} \hat{x}^{m} \int_{\partial\left(U \cap \mathbb{C}_{\mathrm{j}}\right)} s^{-1-m+\ell} d s_{\mathrm{j}} a=\hat{x}^{\ell} a
$$

and this completes the proof. 
The following result is adapted for Clifford numbers from the functional calculus for paravector operators.

Theorem 3.11 (Compactness of the $S$-spectrum). Let $\hat{x} \in \mathbb{R}_{n}$. The $S$-spectrum $\sigma_{S}(\hat{x})$ of $\hat{x}$ is a nonempty, compact set contained in the closed ball $\overline{B_{|\hat{x}|_{1}}(0)}$ of radius $|\hat{x}|_{1}$ and centered at the origin.

Proof. The series $S_{L}^{-1}(s, \hat{x})=\sum_{m=0}^{+\infty} \hat{x}^{m} s^{-m-1}$ converges uniformly on $\partial B_{r}(0)$ for $|\hat{x}|_{1}<r$. For any fixed $\mathrm{j} \in \mathbb{S}$, we have

$$
\int_{\partial\left(B_{r}(0) \cap \mathbb{C}_{\mathrm{j}}\right)} S_{L}^{-1}(s, \hat{x}) d s_{\mathrm{j}}=\sum_{m=0}^{+\infty} \hat{x}^{m} \int_{\partial\left(B_{r}(0) \cap \mathbb{C}_{\mathrm{j}}\right)} s^{-m-1} d s_{\mathrm{j}}=2 \pi,
$$

since it is clear that $\int_{\partial\left(B_{r}(0) \cap \hat{C}_{\mathrm{j}}\right)} s^{-m-1} d s_{\mathrm{j}}$ equals $2 \pi$ if $m=0$ and 0 otherwise. If $\overline{B_{r}(0)}$ was a subset of $\rho_{S}(\hat{x})$, then $S_{L}^{-1}(s, \hat{x})$ would be right slice monogenic on $\overline{B_{r}(0)}$ by Lemma [3.9. Cauchy's integral theorem would then imply that the integral in (3.7) vanishes. However, it is obviously not the case, so we deduce that $\overline{B_{r}(0)} \not \subset \rho_{S}(\hat{x})$ and in turn $\emptyset \neq \sigma_{S}(\hat{x}) \cap \overline{B_{r}(0)}$. This fact implies that $\sigma_{S}(\hat{x})$ is not empty. Let us consider $\mathbb{R}_{n}$ as left (or right) module over itself and let $\mathcal{L}\left(\mathbb{R}_{n}\right)$ be the set of left (or right) linear operators from the Clifford algebra $\mathbb{R}_{n}$ to itself. We consider $\mathcal{L}\left(\mathbb{R}_{n}\right)$ as a real Banach algebra, where the multiplication of a linear operator by a scalar is performed on $\mathbb{R}$. The set $\operatorname{Inv}\left(\mathcal{L}\left(\mathbb{R}_{n}\right)\right)$ of invertible elements of this real Banach algebra is open. Since $\tau: s \mapsto \hat{x}^{2}-2 \operatorname{Re}(s) \hat{x}+|s|^{2}$ is a continuous function with values in $\mathcal{L}\left(\mathbb{R}_{n}\right)$, we deduce that $\rho_{S}(\hat{x})=\tau^{-1}\left(\operatorname{Inv}\left(\mathcal{L}\left(\mathbb{R}_{n}\right)\right)\right)$ is open in $\mathbb{R}_{n}$, so $\sigma_{S}(\hat{x})$ is closed. Theorem 3.3 implies $|s| \leq|\hat{x}|_{1}$ for any $s \in \sigma_{S}(\hat{x})$ and so $\sigma_{S}(\hat{x})$ is a closed subset of the compact set $\overline{B_{|\hat{x}|_{1}}(0)}$ and therefore it is compact.

Theorem 3.12. The integrals (3.8) and (3.9) depend neither on $U$ nor on the imaginary unit $\mathrm{j} \in \mathbb{S}$.

Proof. The independence from the opens set is standard. We just consider (3.8); the other case is similar. The major point in this proof is to show that fully Clifford numbers are such that the integrals are independent of the imaginary unit $\mathrm{j} \in \mathbb{S}$. We show just the crucial points in which we make clear that the replacement of the paravector $x$ by the Clifford number $\hat{x}$ does not invalidate the proof that holds for bounded linear paravector operators.

In order to show the independence of the imaginary unit, we choose two units $\mathrm{i}, \mathrm{j} \in \mathbb{S}$ and two slice Cauchy domains $U_{q}, U_{s} \subset \operatorname{dom}(f)$ with $\sigma_{S}(\hat{x}) \subset U_{q}$ and $\overline{U_{q}} \subset U_{s}$. (The subscripts $q$ and $s$ are chosen in order to indicate the respective variable of integration in the following computation.) The set $U_{q}^{c}:=\mathbb{R}^{n+1} \backslash U_{q}$ is then an unbounded axially symmetric Cauchy domain with $\overline{U_{q}^{c}} \subset \rho_{S}(\hat{x})$. The left $S$-resolvent function is right slice hyperholomorphic on $\rho_{S}(\hat{x})$ and also at infinity because

$$
\lim _{s \rightarrow \infty} S_{L}^{-1}(s, \hat{x})=\lim _{s \rightarrow \infty} \sum_{n=0}^{+\infty} \hat{x}^{n} s^{-n-1}=0 .
$$

The right slice hyperholomorphic Cauchy formula implies therefore

$$
S_{L}^{-1}(s, \hat{x})=\frac{1}{2 \pi} \int_{\partial\left(U_{q}^{c} \cap \mathbb{C}_{\mathrm{i}}\right)} S_{L}^{-1}(q, \hat{x}) d q_{\mathrm{i}} S_{R}^{-1}(q, s)
$$


for any $s \in U_{s}$. As $\partial\left(U_{q}^{c} \cap \mathbb{C}_{\mathrm{j}}\right)=-\partial\left(U_{q} \cap \mathbb{C}_{\mathrm{j}}\right)$ and $S_{R}^{-1}(q, s)=-S_{L}^{-1}(s, q)$, we therefore find

$$
\begin{aligned}
f(\hat{x}) & =\frac{1}{2 \pi} \int_{\partial\left(U_{s} \cap \mathbb{C}_{\mathrm{j}}\right)} S_{L}^{-1}(s, \hat{x}) d s_{\mathrm{j}} f(s) \\
& =\frac{1}{(2 \pi)^{2}} \int_{\partial\left(U_{s} \cap \mathbb{C}_{\mathrm{j}}\right)}\left(\int_{\partial\left(U_{q}^{c} \cap \mathbb{C}_{\mathrm{i}}\right)} S_{L}^{-1}(q, \hat{x}) d q_{\mathrm{i}} S_{R}^{-1}(q, s)\right) d s_{\mathrm{j}} f(s) \\
& =\frac{1}{(2 \pi)^{2}} \int_{\partial\left(U_{q} \cap \mathbb{C}_{\mathrm{i}}\right)} S_{L}^{-1}(q, \hat{x}) d q_{\mathrm{i}}\left(\int_{\partial\left(U_{s} \cap \mathbb{C}_{\mathrm{j}}\right)} S_{L}^{-1}(s, q) d s_{\mathrm{j}} f(s)\right) \\
& =\frac{1}{2 \pi} \int_{\partial\left(U_{q} \cap \mathbb{C}_{\mathrm{i}}\right)} S_{L}^{-1}(q, \hat{x}) d q_{\mathrm{i}} f(q),
\end{aligned}
$$

where the last identity follows again from the slice hyperholomorphic Cauchy formula because we chose $\overline{U_{q}} \subset U_{s}$.

Thanks to Theorem 3.12 Definition 3.13 is well posed.

Definition 3.13 (Slice monogenic functions of a Clifford variable). Let $W \subset \mathbb{R}_{n}$ be a bounded set and let $U \subset \mathbb{R}^{n+1}$ be a bounded slice Cauchy domain that contains $\sigma_{S}(\hat{x})$ for all $\hat{x} \in W$. For $\mathrm{j} \in \mathbb{S}$ we set $d s_{\mathrm{j}}=d s(-\mathrm{j})$.

(I) Assume that $f$ is a (left) slice monogenic function on a set that contains $\bar{U}$. We define the (left) slice monogenic function of the Clifford variable $\hat{x}$ as

$$
f(\hat{x}):=\frac{1}{2 \pi} \int_{\partial\left(U \cap \mathbb{C}_{\mathrm{j}}\right)} S_{L}^{-1}(s, \hat{x}) d s_{\mathrm{j}} f(s) .
$$

(II) Assume that $f$ is a right slice monogenic function on a set that contains $\bar{U}$. We define the (right) slice monogenic function of the Clifford variable $\hat{x}$ as

$$
f(\hat{x}):=\frac{1}{2 \pi} \int_{\partial\left(U \cap \mathbb{C}_{\mathrm{j}}\right)} f(s) d s_{\mathrm{j}} S_{R}^{-1}(s, \hat{x}) .
$$

The next result shows that Definition 3.13 is consistent with polynomials and powers series expansions of slice monogenic functions where we formally replace the paravector variable $x$ by the Clifford variable $\hat{x}$.

Lemma 3.14. Let $\hat{x} \in \mathbb{R}_{n}$. Let us consider the left slice monogenic function $f(s)=\sum_{m=0}^{+\infty} s^{m} p_{m}$ where $p_{m} \in \mathbb{R}_{n}$ converging on $U$ and such that $\sigma_{S}(\hat{x}) \subset U$. Then we have

$$
f(\hat{x})=\sum_{m=0}^{+\infty} \hat{x}^{m} p_{m} .
$$

If the function $f$ is right slice monogenic, i.e., $f(s)=\sum_{m=0}^{+\infty} p_{m} s^{m}$ where $p_{m} \in \mathbb{R}_{n}$ then we have

$$
f(\hat{x})=\sum_{m=0}^{+\infty} p_{m} \hat{x}^{m} .
$$

Proof. Consider the case of left slice monogenic functions. For a suitable $R>0$ the series $\sum_{m=0}^{+\infty} s^{m} p_{m}$ converges in a ball $B(0, R)$ that contains $\sigma_{S}(\hat{x})$. So we can choose another ball

$$
B_{\varepsilon}:=\left\{s \in \mathbb{R}^{n+1}:|s| \leq|\hat{x}|_{1}+\varepsilon\right\}
$$


for sufficiently small $\varepsilon>0$, such that $B_{\varepsilon} \subset B(0, R)$. Since the series converges uniformly on $\partial B_{\varepsilon}$ we have

$$
\begin{aligned}
f(\hat{x}) & =\frac{1}{2 \pi} \int_{\partial\left(B_{\varepsilon} \cap \mathbb{C}_{\mathrm{j}}\right)} S_{L}^{-1}(s, \hat{x}) d s_{\mathrm{j}} \sum_{m=0}^{+\infty} s^{m} p_{m} \\
& =\frac{1}{2 \pi} \sum_{m=0}^{+\infty} \int_{\partial\left(B_{\varepsilon} \cap \mathbb{C}_{\mathrm{j}}\right)} S_{L}^{-1}(s, \hat{x}) d s_{\mathrm{j}} s^{m} p_{m} \\
& =\frac{1}{2 \pi} \sum_{m=0}^{+\infty} \int_{\partial\left(B_{\varepsilon} \cap \mathbb{C}_{\mathrm{j}}\right)} \sum_{k=0}^{+\infty} \hat{x}^{k} s^{-1-k} d s_{\mathrm{j}} s^{m} p_{m}=\sum_{m=0}^{+\infty} \hat{x}^{m} p_{m} .
\end{aligned}
$$

The case of right slice monogenic functions is the same with the obvious changes.

Remark 3.15. We point out that in the definition of slice monogenic functions of a Clifford variable we made two choices: we fixed a Clifford algebra $\mathbb{R}_{n}$ and we used slice monogenic functions defined on an open set in the Euclidean space $\mathbb{R}^{n+1}$. The $S$-spectrum of $\hat{x} \in \mathbb{R}_{n}$ is a subset of $\mathbb{R}^{n+1}$ and so it depends on the latter choice. We could have defined the $S$-spectrum as subset of $\mathbb{R}^{m+1}$, with $m \leq n$, and used slice monogenic functions defined on open sets in $\mathbb{R}^{m+1}$ (see [9]). The choice $m=n$ corresponds to the maximal Euclidean space that can be used as domain of slice monogenic functions.

Since slice monogenic functions of a Clifford variable are defined via a functional calculus the product of two of such functions is well defined when we have the product rule for the functional calculus. In order to do this we recall that the $S$ resolvent functions satisfy the $S$-resolvent equation, as it can be checked by a direct computation. We have the following results.

Theorem 3.16. Let $\hat{x} \in \mathbb{R}_{n}$ and let $s \in \rho_{S}(\hat{x})$. The left $S$-resolvent function satisfies the left $S$-resolvent equation

$$
S_{L}^{-1}(s, \hat{x}) s-\hat{x} S_{L}^{-1}(s, \hat{x})=1
$$

and the right $S$-resolvent function satisfies the right $S$-resolvent equation

$$
s S_{R}^{-1}(s, \hat{x})-S_{R}^{-1}(s, \hat{x}) \hat{x}=1 .
$$

The left and the right $S$-resolvent equations cannot be considered the generalization of the classical resolvent equation. The $S$-resolvent equation entangles the $S$-resolvent functions and the slice monogenic Cauchy kernel in the following way.

Theorem 3.17 (The $S$-resolvent equation [1]). Let $\hat{x} \in \mathbb{R}_{n}$ and let $s, q \in \rho_{S}(\hat{x})$ with $q \notin[s]$. Then the equation

$$
\begin{aligned}
S_{R}^{-1}(s, \hat{x}) S_{L}^{-1}(q, \hat{x})= & {\left[\left(S_{R}^{-1}(s, \hat{x})-S_{L}^{-1}(q, \hat{x})\right) q\right.} \\
& \left.-\bar{s}\left(S_{R}^{-1}(s, \hat{x})-S_{L}^{-1}(q, \hat{x})\right)\right]\left(q^{2}-2 \operatorname{Re}(s) q+|s|^{2}\right)^{-1}
\end{aligned}
$$

holds true. Equivalently, it can also be written as

$$
\begin{aligned}
S_{R}^{-1}(s, \hat{x}) S_{L}^{-1}(q, \hat{x}) & =\left(s^{2}-2 \operatorname{Re}(q) s+|q|^{2}\right)^{-1} \\
\cdot & {\left[\left(S_{L}^{-1}(q, \hat{x})-S_{R}^{-1}(s, \hat{x})\right) \bar{q}-s\left(S_{L}^{-1}(q, \hat{x})-S_{R}^{-1}(s, \hat{x})\right)\right] . }
\end{aligned}
$$

As a consequence of the $S$-resolvent equation we obtain the product rule. 
Theorem 3.18 (Product rule). Let $\hat{x} \in \mathbb{R}_{n}$ and let $f \in \mathcal{N}\left(\sigma_{S}(\hat{x})\right)$ and $g \in$ $\mathcal{S M}_{L}\left(\sigma_{S}(\hat{x})\right)$ or let $f \in \mathcal{S M}_{R}\left(\sigma_{S}(\hat{x})\right)$ and $g \in \mathcal{N}\left(\sigma_{S}(\hat{x})\right)$. Then

$$
(f g)(\hat{x})=f(\hat{x}) g(\hat{x}) .
$$

Proof. It is a consequence of the $S$-resolvent equation and of the relation

$$
\frac{1}{2 \pi} \int_{\partial\left(U \cap \mathbb{C}_{\mathrm{j}}\right)} f(s) d s_{\mathrm{j}}(\bar{s} \hat{x}-\hat{x} q)\left(q^{2}-2 \operatorname{Re}(s) q+|s|^{2}\right)^{-1}=\hat{x} f(q)
$$

that holds true if $f$ is an intrinsic slice hyperholomorphic function and $U$ is a bounded slice Cauchy domain with $\bar{U} \subset \operatorname{dom}(f)$ for any $q \in U$ and any $\mathrm{j} \in \mathbb{S}$.

Theorem 3.19 can be proved following the proof in the case of paravector operators and so we omit the proof.

Theorem 3.19. The following facts hold.

(I) (The spectral mapping theorem) Let $\hat{x} \in \mathbb{R}_{n}$ and let $f \in \mathcal{N}\left(\sigma_{S}(\hat{x})\right)$. Then

$$
\sigma_{S}(f(\hat{x}))=f\left(\sigma_{S}(\hat{x})\right)=\left\{f(s): s \in \sigma_{S}(\hat{x})\right\} .
$$

(II) (Spectral radius theorem) Let $\hat{x} \in \mathbb{R}_{n}$; then the $S$-spectral radius of $\hat{x}$ is defined to be the nonnegative real number

$$
r_{S}(\hat{x}):=\sup \left\{|s|: s \in \sigma_{S}(\hat{x})\right\} .
$$

Then for $\hat{x} \in \mathbb{R}_{n}$, we have

$$
r_{S}(\hat{x})=\lim _{m \rightarrow+\infty}\left|\hat{x}^{m}\right|_{1}^{\frac{1}{m}} .
$$

(III) (Composition rule) Let $\hat{x} \in \mathbb{R}_{n}$ and let $f \in \mathcal{N}\left(\sigma_{S}(\hat{x})\right)$. If $g \in \mathcal{S} \mathcal{M}_{L}\left(\sigma_{S}(f(\hat{x}))\right.$ then $g \circ f \in \mathcal{S M}_{L}\left(\sigma_{S}(\hat{x})\right)$ and if $g \in \mathcal{S M}_{R}\left(f\left(\sigma_{S}(\hat{x})\right)\right)$ then $g \circ f \in \mathcal{S H}_{R}\left(\sigma_{S}(\hat{x})\right)$. In both cases

$$
g(f(\hat{x}))=(g \circ f)(\hat{x}) .
$$

\section{Further consequences of the $S$-Functional Calculus}

In the preceding sections, we discussed how the use of operator theory allows to extend the definition of slice monogenic functions from paravectors to all the elements in a Clifford algebra. But this may go beyond Clifford numbers. We now give further examples to illustrate the advantages of our method based on the $S$-functional calculus.

4.1. Composition of slice monogenic functions with monogenic functions. The definition of slice monogenic functions of a Clifford variable has important implications in the function theory of monogenic functions because it allows to define the composition of a slice monogenic function with a monogenic function. This composition is otherwise undefined between these functions. In fact, let $U_{0}$ be an open set and let $\breve{f}: U_{0} \subseteq \mathbb{R}^{n+1} \rightarrow \mathbb{R}_{n}$ be a monogenic function. We determine the $S$-spectrum of $\breve{f}(x)$

$$
\sigma_{S}(\breve{f}(x))=\left\{s \in \mathbb{R}^{n+1}: \breve{f}^{2}(x)-2 \operatorname{Re}(s) \breve{f}(x)+|s|^{2} \text { is not invertible in } \mathbb{R}_{n}\right\}
$$

and, given the slice monogenic function $f$ defined on an axially symmetric domain $U$ which contains $\sigma_{S}(\breve{f}(x))$, we define the composition $f(\breve{f})(x)$ as

$$
f(\breve{f})(x):=\frac{1}{2 \pi} \int_{\partial\left(U \cap \mathbb{C}_{j}\right)} S_{L}^{-1}(s, \breve{f}(x)) d s_{j} f(s) .
$$


One has to pay attention also on the dependence on $x$ in the definition of the $S$-spectrum. This fact has many profound consequences on the function theory.

4.2. Slice monogenic functions of an octonionic variable. Let $\mathbb{O}$ be the noncommutative and nonassociative division algebra of octonions. We define the $S$ spectrum associated with an octonionic number as follows:

Definition 4.1 (The $S$-spectrum of an octonion). Let $Q \in \mathbb{O}$; we can define various notions of spectrum, according to the choice of a set $S$ : We define the $S$-spectrum of the octonionic number $Q \in \mathbb{O}$ as

$$
\sigma_{S}(Q)=\left\{s \in \mathcal{S}: Q^{2}-2 \operatorname{Re}(s) Q+|s|^{2} \text { is not invertible in } \mathbb{O}\right\} .
$$

We can obviously choose $\mathcal{S}=\mathbb{O}$ but other cases are possible. With $\mathcal{S}=\mathbb{H}$, we have a quaternionic spectrum of an octonion and we can consider the functional calculus for slice hyperholomorphic functions of a quaternionic variable and with quaternionic values, thus obtaining that $f(Q)$ is a function with values in $\mathbb{O}_{\mathbb{H}}:=$ $\mathbb{O} \otimes \mathbb{H}$,

In principle, we could also consider the algebraic tensor product $\mathbb{O} \otimes \mathbb{R}_{n}$ over the reals of $\mathbb{O}$ with the Clifford algebra $\mathbb{R}_{n}$ and we set $\mathbb{O}_{n}:=\mathbb{O} \otimes \mathbb{R}_{n}$. Using the $S$-functional calculus we can now define slice monogenic functions (with coefficients in $\mathbb{R}_{n}$ ) of an octonionic variable and with values in $\mathbb{O}_{n}$.

We use the $S$-resolvent functions and the $S$-functional calculus defined for slice hyperholomorphic functions (with quaternionic coefficients) of an octonionic variable.

It would be interesting to investigate possible extensions of the results in [26, 28] according to this new definitions. We will not pursue this here.

4.3. Noncommuting matrix variables. As another example, we consider the case of $(n+1)$ noncommuting matrices. Precisely let $X_{j} \in \mathbb{R}^{d \times d}$, for $d \in \mathbb{N}$, and fix a Clifford algebra $\mathbb{R}_{n}$. We make the identification

$$
\left(X_{0}, X_{1}, \ldots, X_{n}\right) \rightarrow \mathbf{X}=\sum_{j=0}^{n} X_{j} e_{j}
$$

to identify $(n+1)$-tuples of $d \times d$ real matrices with a $d \times d$ matrix with paravector entries. The $S$-spectrum of the $(n+1)$-tuple of noncommuting matrices $\left(X_{0}, X_{1}, \ldots, X_{n}\right)$ is defined as:

Definition 4.2. Let $\mathbf{X}=\sum_{j=1}^{n} X_{j} e_{j}$, and take $s \in \mathbb{R}^{n+1}$. We define the $S$ spectrum of the $\mathbf{X} \in \mathbb{R}^{d \times d}$ as

$$
\sigma_{S}(\mathbf{X})=\left\{s \in \mathbb{R}^{n+1}: \mathbf{X}^{2}-2 \operatorname{Re}(s) \mathbf{X}+|s|^{2} \mathcal{I}_{d \times d} \text { is not invertible in } \mathbb{R}^{d \times d}\right\}
$$

and the $S$-resolvent set as

$$
\rho_{S}(\mathbf{X})=\mathbb{R}^{n+1} \backslash \sigma_{S}(\mathbf{X}) .
$$

Note that we can consider $2^{n}$-tuples of matrices identified with $\mathbf{X}=\sum_{|A|=0}^{n} X_{A} e_{A}$ whose $S$-spectrum $\sigma_{S}(\mathbf{X})$ is given above. This approach may give more useful properties on the operator $\mathbf{X}$. Moreover, the $S$-resolvent functions keep the same form: 
Definition 4.3. Let $\mathbf{X} \in \mathbb{C}^{d \times d} \otimes \mathbb{R}_{n}$. For $s \in \rho_{S}(\mathbf{X})$, we define the left $S$-resolvent function as

$$
S_{L}^{-1}(s, \mathbf{X})=-\left(\mathbf{X}^{2}-2 \operatorname{Re}(s) \mathbf{X}+|s|^{2} \mathcal{I}_{d \times d}\right)^{-1}\left(\mathbf{X}-\bar{s} \mathcal{I}_{d \times d}\right),
$$

and the right $S$-resolvent function as

$$
S_{R}^{-1}(s, \mathbf{X})=-\left(\mathbf{X}-\bar{s} \mathcal{I}_{d \times d}\right)\left(\mathbf{X}^{2}-2 \operatorname{Re}(s) \mathbf{X}+|s|^{2} \mathcal{I}_{d \times d}\right)^{-1} .
$$

Via the $S$-functional calculus we can define slice monogenic functions (with coefficients in $\mathbb{R}_{n}$ ) of the noncommuting matrices $\mathbf{X}$. In particular the case of intrinsic functions contains all special functions that have power series expansion like the exponential, sine, cosine, Bessel, more in general hypergeometric functions to name a few.

\section{ACKNOWLEDGMENTS}

The authors would like to thank both the referees for carefully reading the manuscript and for their comments.

\section{REFERENCES}

[1] Daniel Alpay, Fabrizio Colombo, Jonathan Gantner, and Irene Sabadini, A new resolvent equation for the S-functional calculus, J. Geom. Anal. 25 (2015), no. 3, 1939-1968, DOI 10.1007/s12220-014-9499-9. MR 3358079

[2] Daniel Alpay, Fabrizio Colombo, Tao Qian, and Irene Sabadini, The $H^{\infty}$ functional calculus based on the $S$-spectrum for quaternionic operators and for $n$-tuples of noncommuting operators, J. Funct. Anal. 271 (2016), no. 6, 1544-1584, DOI 10.1016/j.jfa.2016.06.009. MR 3530583

[3] Fred Brackx, Richard Delanghe, and Franciscus Sommen, Clifford analysis, Research Notes in Mathematics, vol. 76, Pitman (Advanced Publishing Program), Boston, MA, 1982. MR697564

[4] Fabrizio Colombo and Jonathan Gantner, Quaternionic closed operators, fractional powers and fractional diffusion processes, Operator Theory: Advances and Applications, vol. 274, Birkhäuser/Springer, Cham, 2019, DOI 10.1007/978-3-030-16409-6. MR3967697

[5] Fabrizio Colombo, Jonathan Gantner, and David P. Kimsey, Spectral theory on the Sspectrum for quaternionic operators, Operator Theory: Advances and Applications, vol. 270, Birkhäuser/Springer, Cham, 2018. MR3887616

[6] Fabrizio Colombo, Jonthan Gantner, David P. Kimsey, and Irene Sabadini, Universality property of the $S$-functional calculus, noncommuting matrix variables and Clifford operators, Preprint, 2020.

[7] Fabrizio Colombo and David P. Kimsey, The spectral theorem for normal operators on a Clifford module, Preprint, 2020.

[8] Fabrizio Colombo, Rolf Sören Kraußhar, and Irene Sabadini, Symmetries of slice monogenic functions, J. Noncommut. Geom. 14 (2020), no. 3, 1075-1106, DOI 10.4171/jncg/387. MR 4170649

[9] Fabrizio Colombo, Rolf Sören Kraußhar, and Irene Sabadini, Slice monogenic theta series, Preprint, 2021.

[10] Fabrizio Colombo, Roman Lávička, Irene Sabadini, and Vladim Souček, The Radon transform between monogenic and generalized slice monogenic functions, Math. Ann. 363 (2015), no. 34, 733-752, DOI 10.1007/s00208-015-1182-3. MR3412341

[11] Fabrizio Colombo and Irene Sabadini, A structure formula for slice monogenic functions and some of its consequences, Hypercomplex analysis, Trends Math., Birkhäuser Verlag, Basel, 2009, pp. 101-114. MR2742644

[12] Fabrizio Colombo and Irene Sabadini, The Cauchy formula with s-monogenic kernel and a functional calculus for noncommuting operators, J. Math. Anal. Appl. 373 (2011), no. 2, 655-679, DOI 10.1016/j.jmaa.2010.08.016. MR2720712

[13] Fabrizio Colombo, Irene Sabadini, and Daniele C. Struppa, Slice monogenic functions, Israel J. Math. 171 (2009), 385-403, DOI 10.1007/s11856-009-0055-4. MR2520116 
[14] Fabrizio Colombo, Irene Sabadini, and Daniele C. Struppa, An extension theorem for slice monogenic functions and some of its consequences, Israel J. Math. 177 (2010), 369-389, DOI 10.1007/s11856-010-0051-8. MR2684426

[15] Fabrizio Colombo, Irene Sabadini, and Daniele C. Struppa, Noncommutative functional calculus, Progress in Mathematics, vol. 289, Birkhäuser/Springer Basel AG, Basel, 2011. Theory and applications of slice hyperholomorphic functions, DOI 10.1007/978-3-0348-0110-2. MR 2752913

[16] Fabrizio Colombo, Irene Sabadini, and Daniele C. Struppa, A new functional calculus for noncommuting operators, J. Funct. Anal. 254 (2008), no. 8, 2255-2274, DOI 10.1016/j.jfa.2007.12.008. MR2402108

[17] Fabrizio Colombo, Irene Sabadini, Franciscus Sommen, and Daniele C. Struppa, Analysis of Dirac systems and computational algebra, Progress in Mathematical Physics, vol. 39, Birkhäuser Boston, Inc., Boston, MA, 2004, DOI 10.1007/978-0-8176-8166-1. MR2089988

[18] Lander Cnudde, Hendrik De Bie, and Guangbin Ren, Algebraic approach to slice monogenic functions, Complex Anal. Oper. Theory 9 (2015), no. 5, 1065-1087, DOI 10.1007/s11785014-0393-z. MR3346770

[19] Jonathan Gantner, A direct approach to the $S$-functional calculus for closed operators, J. Operator Theory 77 (2017), no. 2, 287-331, DOI 10.7900/jot.2017mar24.2092. MR.3634508

[20] Graziano Gentili and Daniele C. Struppa, A new approach to Cullen-regular functions of a quaternionic variable (English, with English and French summaries), C. R. Math. Acad. Sci. Paris 342 (2006), no. 10, 741-744, DOI 10.1016/j.crma.2006.03.015. MR.2227751

[21] Graziano Gentili and Daniele C. Struppa, Regular functions on the space of Cayley numbers, Rocky Mountain J. Math. 40 (2010), no. 1, 225-241, DOI 10.1216/RMJ-2010-40-1-225. MR2607115

[22] Riccardo Ghiloni and Alessandro Perotti, Slice regular functions on real alternative algebras, Adv. Math. 226 (2011), no. 2, 1662-1691, DOI 10.1016/j.aim.2010.08.015. MR2737796

[23] Riccardo Ghiloni and Vincenzo Recupero, Semigroups over real alternative *-algebras: generation theorems and spherical sectorial operators, Trans. Amer. Math. Soc. 368 (2016), no. 4, 2645-2678, DOI 10.1090/tran/6399. MR3449252

[24] Brian Jefferies, Spectral properties of noncommuting operators, Lecture Notes in Mathematics, vol. 1843, Springer-Verlag, Berlin, 2004. MR2069293

[25] Ming Jin and Guangbin Ren, Cauchy kernel of slice Dirac operator in octonions with complex spine, Complex Anal. Oper. Theory 14 (2020), no. 1, Paper No. 17, 24, DOI 10.1007/s11785019-00977-0. MR 4056946

[26] Ming Jin, Guangbin Ren, and Irene Sabadini, Slice Dirac operator over octonions, Israel J. Math. 240 (2020), no. 1, 315-344, DOI 10.1007/s11856-020-2067-z. MR4193136

[27] Pierce Wadell Ketchum, Analytic functions of hypercomplex variables, Trans. Amer. Math. Soc. 30 (1928), no. 4, 641-667, DOI 10.2307/1989440. MR1501452

[28] Rolf Sören Kraußhar, Differential topological aspects in octonionic monogenic function theory, Adv. Appl. Clifford Algebr. 30 (2020), no. 4, Paper No. 51, 25, DOI 10.1007/s00006020-01074-8. MR 4130131

[29] M. Elena Luna-Elizarrarás, Michael Shapiro, Daniele C. Struppa, and Adrian Vajiac, Bicomplex holomorphic functions, Frontiers in Mathematics, Birkhäuser/Springer, Cham, 2015. The algebra, geometry and analysis of bicomplex numbers, DOI 10.1007/978-3-319-24868-4. MR3410909

[30] Guangbin Ren and Xieping Wang, Growth and distortion theorems for slice monogenic functions, Pacific J. Math. 290 (2017), no. 1, 169-198, DOI 10.2140/pjm.2017.290.169. MR 3673083

[31] Giovanni Battista Rizza, Funzioni regolari nelle algebre di Clifford (Italian), Rend. Mat. e Appl. (5) 15 (1956), 53-79. MR83546

[32] Michele Sce, Monogeneità e totale derivabilità nelle algebre reali e complesse. I (Italian), Atti Accad. Naz. Lincei Rend. Cl. Sci. Fis. Mat. Nat. (8) 16 (1954), 30-35. MR67851 
Dipartimento di Matematica, Politecnico di Milano, Via E. Bonardi, 9, 20133 Milano, ITALY

Email address: fabrizio.colombo@polimi.it

School of Mathematics, Statistics and Physics, Newcastle University, Newcastle Upon Tyne NE1 7RU, United Kingdom

Email address: david.kimsey@ncl.ac.uk

Dipartimento di Matematica, Politecnico di Milano, Via E. Bonardi, 9, 20133 Milano, ITALY

Email address: stefano.pinton@polimi.it

Dipartimento di Matematica, Politecnico di Milano, Via E. Bonardi, 9, 20133 Milano, ITALY

Email address: irene.sabadini@polimi.it 